\title{
Phaechromacytoma Associated with Fatal Myocardial Infarction
}

\author{
Shirley Siew ${ }^{1 *}$ and James Richards ${ }^{2}$ \\ 1. Human Pathology, Division of Human Pathology, College of Osteopathic Medicine, Michigan State \\ University, East Lansing, Michigan, USA. \\ 2. Department of Pathology, E. W. Sparrow Hospital, Lansing, Michigan, USA. \\ * Corresponding author: shirley.siew@hc.msu.edu
}

Pheochromocytoma is a rare tumor found in approximately 2 individuals per million of the population. In $90 \%$, it arises from the chromaffin cells of the adrenal medulla, which secrete catecholamines-epinephrine and norepinephrine. It has been called the $10 \%$ tumor - $10 \%$ bilateral, $10 \%$ outside the abdomen, $10 \%$ malignant [1], 10\% in children and people with a family history. The secretion of catecholamines has a sympathomimetic action-producing headache, palpitations excessive sweating, tremulousness, chest pain. A classical presentation is paroxysms of hypertension. The catecholamines are excreted as vanilyl mandelic acid.

Macroscopic features - well-circumscribed, homogenious grey-brown color. Histopathology-Cells tend to be grouped and separated by delicate vascularized septa. Finely granular cytoplasm. Reduce chrome salts resulting in a brown color (Fig.1)

We present a case of a 47-year-old man, who developed a discomfort in the central substernal area of the chest, while he was in church. This was accompanied with nausea, dry heaving, excessive sweating, and a headache. There were episodes of dizziness, confusion and tingling of the face. He was an insulin dependent diabetic and since the previous day he had had extremely elevated blood sugar levels despite a bolus of insulin. About 2pm., he developed labored breathing with wheezing. Boluses of Lopressor were administered in the Emergency Department and he was put onto a heparin and nitroglycerin drip, and, was given sublingual nitro glycerin - basic therapy for myocardial ischemia. Electrocardiogram showed borderline junctional depression and a bedside echocardiogram -showed an inferolateral hypokinesis with an ejection fraction of $40 \%$. The tachycardia and diaphoresis persisted. Laboratory finding were consistent with myocardial infarction with increase of Troporin T-2-80; B-type natriuretic peptide. 237; Creatine phosphokinase -237 ; CK - MB $19.4-8.2 \%$. There was worsening lactic acidosis. The blood pressure rose to $190 / 100 \mathrm{mmHg}$ and $200 / 112 \mathrm{mmHg}$ on recheck; heart rate 140 per minute. The patient went into a cardiac arrest and did not respond to CPR.

Autopsy showed the presence of a pheochromcytoma in the left adrenal; myocardial infarction in the inferolateral portion of the left ventricle, pulmonary edema with foci of hemorrhage.

Histopathology-benign pheochromocytoma (Fig. 1); myocardial infarction 24 hours-Waviness of the myofibers (Fig.2); contraction bands of the miyofibers (Fig 3); early polymorphonuclear cell infiltration (Fig.4).

This case illustrates the clinical presentation of the sympathomimetic effects of catecholamine secretion resulting in critical myocardial ischemia and fatal infarction. 
References:

[1] S Siew, Microsc. Microanalysis. 4 (1998), p.1086.

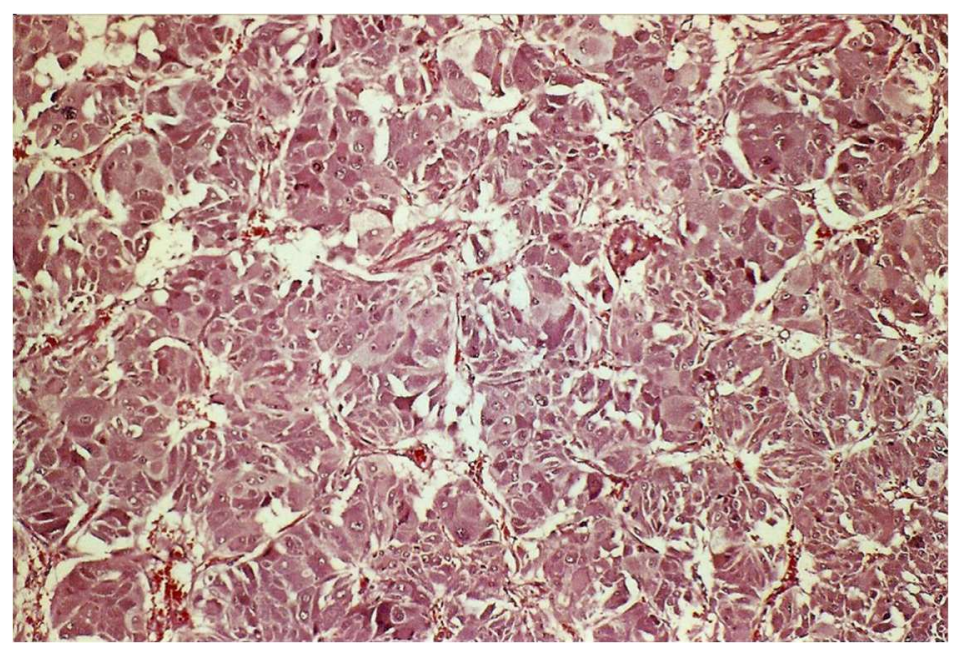

Figure 2. PHE0CHROMACYTOMA original magnification $400 \mathrm{X}$

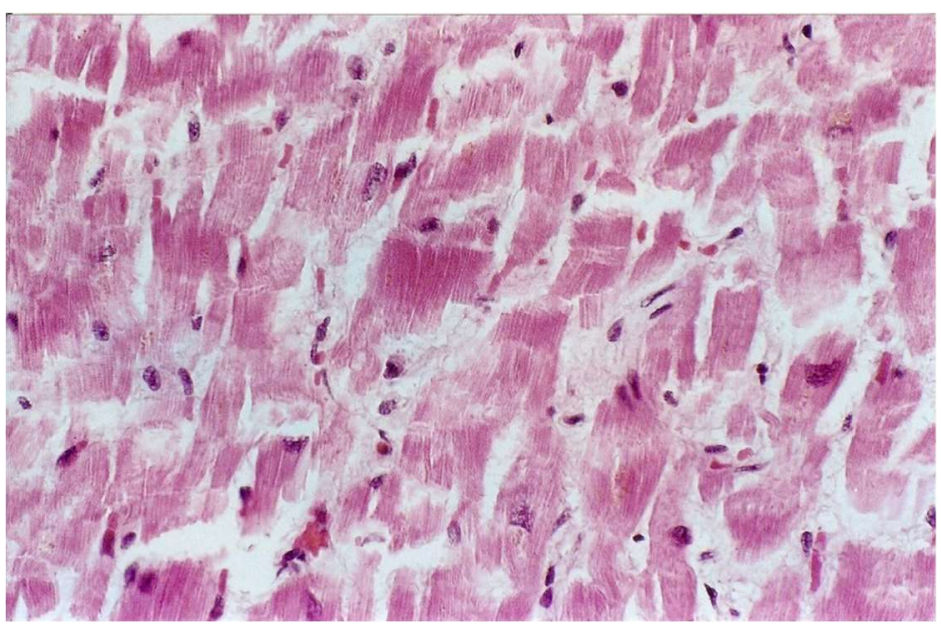

Figure 1. contraction bands of the miyofibers original magnification $400 \mathrm{X}$

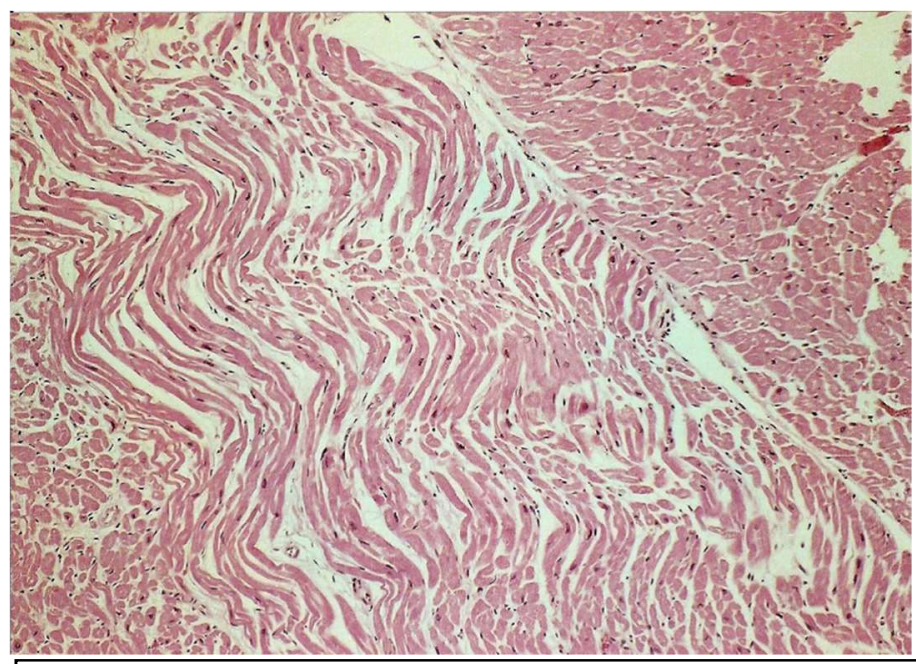

Figure 3. Waviness of the myofibers original magnification 400X

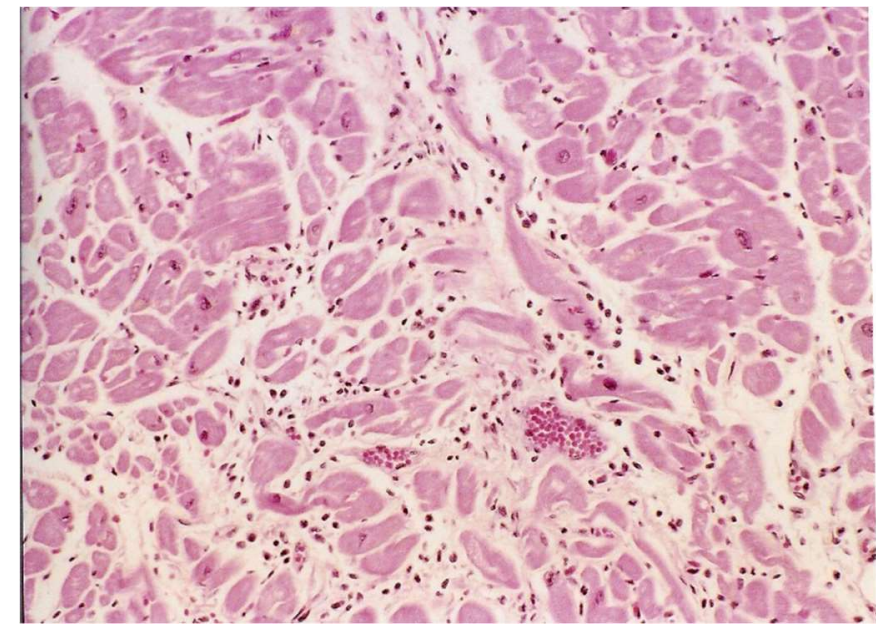

Figure 4. early polymorphonuclear cell infiltration 